\title{
The Critical Temperature of The Superconductor Having an Array of Holes
}

\author{
Harsojo* \\ Department of Physics University Gadjah Mada \\ Sekip Utara, Yogyakarta 55281
}

\begin{abstract}
The influence of external magnetic field to the critical temperature of the superconductor having an array of hole has been calculated using time dependent Ginzburg-Landau equation. The investigation is done after calculating numerically the resistance produced when the superconductor is driven by electric current and exposed under perpendicular magnetic field fie. The result indicates that the addition of lattice holes to the superconductor exposed on perpendicular magnetic field changes the critical temperature. The changes of the critical current due to the different sizes of holes are also discussed.
\end{abstract}

KEYWORDS: superconductor, critical temperature, lattice holes, Ginzburg-Landau equation.

\section{INTRODUCTION}

Lattice holes addition to superconductor has started become a new technique in enhancing the critical current density [1]. Experiment and theoretical confirmation of this technique in enhancing the critical current of superconductor were investigated by many authors [1]-[3]. It was understood that the enhancing of the critical current on superconductor having holes lattice is due to the vortex-hole and vortex-vortex interaction [4]. These interactions produce a pinning force such that in matching magnetic flux between the lattice hole structure and flux quantization it enhances the critical current significantly. The experiment and numerical calculation were observed by some authors such as in [5]-[6]. In matching magnetic fields $H_{n}=n \Phi_{\circ} / A$, where $\mathrm{n}$ may be integer or fractional number and $\Phi_{\circ}$ is unit flux quantization, and $\mathrm{A}$ is the area of a unit lattice hole [1]-[3]. Experimentally, it was pointed out that due to the addition of an array of hole, the critical temperature of the superconductor become higher [1]. It is understood that this critical temperature change in the superconductor having such holes is due to Little-Park effect [5] and it was observed at high Tc superconductor as well [6]. However, observing these phenomena is interesting since such a superconductor configuration may be used for enginering application where the temperature is a control parameter in the interest quantities. When experiment becomes difficult to be performed in predicting the effect of a hole lattice structure to the critical current, a numerical calculation or a simulation becomes important. However, there have only been a few numerical studies are available. Some previous studies along this line are usually done using time independent Ginzburg-landau equation (TIGLE) [6] applied to superconductor having a few hole or molecular dynamics [7]-[8]. In this contribution, instead of using TIGLE, we study the phenomenon using time depen-

*E-MAIL: harsojougmeugm.ac.id dent Ginzburg-Landau equation (TDGL) and apply to superconductor having an array of holes with 2 different sizes both in the order of superconductor coherence length.

\section{MODEL}

\section{A. TDGL equation}

In our calculation, we use the simplest time dependent description, namely TDGL with the scalar order parameter $\Psi$. We assumed that complex scalar order parameter is sufficient to describe the vortex dynamics of the system. The external magnetic field expresses in the form of vector field couple with the scalar order parameter through TDGL equation. The electric potential is assumed to be zero. We use normalized scale to form the TDGL [4],[10] by expressing length unit in coherence length at $0 \mathrm{~K}, \xi(0)$, assuming $\xi(T)=$ $\xi(0)\left(1-T_{c}\right)^{-1 / 2}$

$$
\begin{aligned}
& \partial_{t} \mathbf{A}=-\kappa^{2} \nabla \times \nabla \times \mathbf{A}+(1-T) \operatorname{Re} \Psi^{*}(-i \nabla-A) \Psi(1) \\
& \partial_{t} \Psi=\frac{1}{\eta}(-i \nabla-\mathbf{A})^{2} \Psi+(1-T)\left(|\Psi|^{2}-1\right) \Psi
\end{aligned}
$$

In this form, the super current and the normal current density is written respectively as $\mathbf{J}_{S}=\frac{1}{2}\left(\Psi^{*} \nabla \Psi-\Psi \nabla \Psi^{*}\right)-$ $|\Psi|^{2} \mathbf{A}$ and $\mathbf{J}_{n}=-\partial_{t} \mathbf{A}$. Therefore, the total current density is $\mathbf{J}=\mathbf{J}_{n}+\mathbf{J}_{S}$. To accommodate the existence of holes, we have to imply the proper boundary condition. In our model, the magnetic field is $B_{z}=(\nabla \times \mathbf{A}) \bullet \vec{z}=H$ on the surface of the superconductor. It indicates that the external magnetic field is on the $\mathrm{z}$-direction. The current density flows on the $\mathrm{x}$-direction such that $\widehat{x} \bullet(i \nabla-\mathbf{A}) \Psi=-J_{t}$, while $\widehat{y} \bullet(i \nabla-\mathbf{A}) \Psi=0$ where $\widehat{x}$ and $\widehat{y}$ is unit normal vectors. The boundary condition of $\widehat{y} \bullet(i \nabla-\mathbf{A}) \Psi=0$ is implemented at the edges of each square hole. Inside the holes, the magnetic field $H_{i}$ is equal to external magnetic field $\mathrm{H}$ while at 


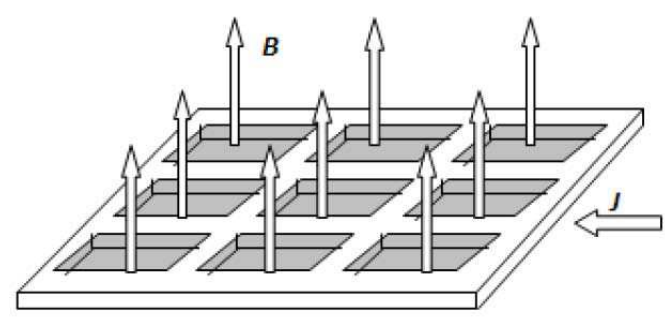

FIG. 1: Model of the superconductor having an arry of holes exposed under the electric current and magnetic field. The size hole is symbolized with $S_{x}$ where x indicates the hole edge size

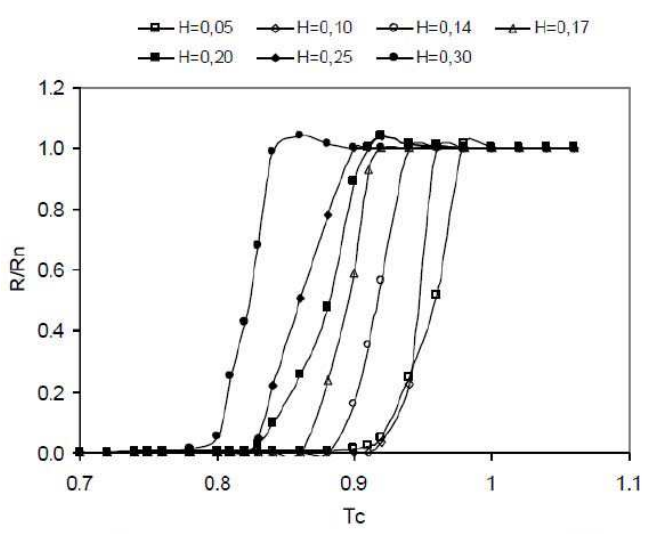

FIG. 2: The resistance versus the temperature on superconductor having lattice parameter of $7.5 \xi(0)$ and hole size of $1.5 \xi(0)$

the boundary between the superconductor and the holes satisfy $B_{z}=H_{i}$. When array of holes added to the superconductor exposed under vertical magnetic field, there exists matching field phenomena in which the total magnetic flux matches with the lattice hole array cell. In this situation, the matching magnetic field are $H_{n}=n \frac{\Phi_{\circ}}{A}$, where $\Phi_{\circ}$ is the flux unit, is the size of the cell, and is an integer or fractional number. In such magnetic fields, vortices will arrange themselves to be stable by sitting at the holes and the interstitials. The first matching field is related to the vortices occupy the holes while the second matching field is related to the condition where the vortices the interstitials. In this way the electric currents at the matching magnetic fields are enhanced. The model is shown at Fig.1.

Having an external current added to the sample, some extra boundary shall be managed such that the first derivative of the normal scalar field zero and the local magnetic field will contain the sum of external magnetic field and the current induced magnetic field. This boundary condition is taken by assuming that the superconductor material is bounded by the normal materials at side edges where the electric current flows. The external current density, $J_{t}$, is introduced via the local magnetic field [11]. In the upper part the induced magnetic field will be added by $-L J_{t} / 2$ while on the lower part it will be added by $L J_{t} / 2$ where $\mathrm{L}$ is the width of the sample. Having

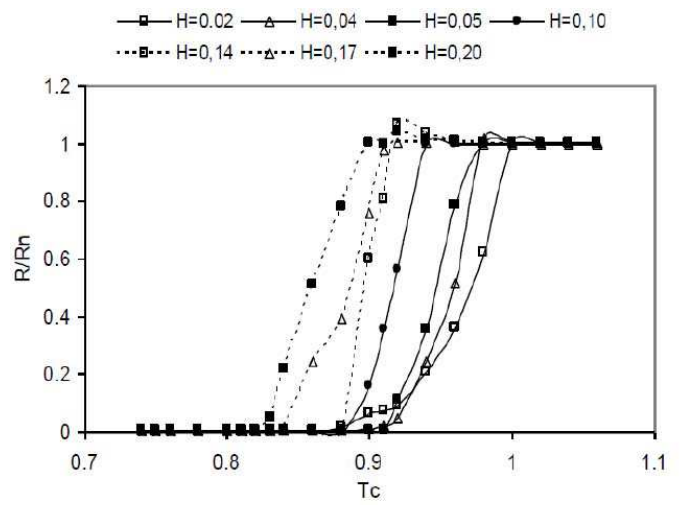

FIG. 3: The resistance versus the temperature on superconductor having lattice parameter of $7.5 \xi(0)$ and hole size of $2.5 \xi(0)$

this done in two dimensions approach and using finite difference approximation for space and using linear integration in time [4], we can calculate directly the voltage produced when the electric current flows at the superconductor. We select a small electric current $(j=0.0001)$ applied on the superconductor such that the vortices move producing electric voltage from which the resistance can be calculated. Since we assumed no electric potential is supplied, the resistance here is therefore due to the vortices motion because of the Lorentz force. Similar situation happens when the electric current is applied to the superconductor and exposed under an external magnetic field $\mathrm{H}$ [4]. Finally, knowing the electric current and the electric voltage, the resistance $\mathrm{R}(\mathrm{H})$ can then be calculated. The calculation of $\mathrm{R}(\mathrm{H})$ is done for various temperature to obtain $\mathrm{R}(\mathrm{H}, \mathrm{T})$ by still keeping the validity of the Ginzburg-Landau approximation for $T / T_{c}>0,5$. Changes of the critical temperature $\Delta T_{c}$ related to the superconductor having hole radius $\mathrm{R}$ and area $\mathrm{S}$ exposed under external magnetic field $\mathrm{H}$ as

$$
\frac{\Delta T_{c}}{T_{c}}=\left(\frac{\xi_{\circ}}{R}\right)^{2}\left(n-\frac{H S}{\Phi_{\circ}}\right)^{2}
$$

where $n$ integer number. Since the total number of vortices always bigger then the number of vortices trapping in the hole, therefore $\Delta T_{c}>0$. To calculate $\Delta T_{c}$ the boundary conditions on $\mathrm{N}$ holes must also be supplied accordingly. We use superconductor having $\kappa=2$. The mesh size of the superconductor is $0.5 \xi(0)$, so that the calculation was done in $N^{2}$ meshes array while is the number of meshes related to the size of superconductor. The proper boundary must be matched since the position of $\Psi$ is different with the position of $\mathbf{A}$.

\section{RESULT AND DISCUSSION}

The calculation was done on superconductor having lattice constant $0.75 \xi(0)$, number of holes are 9 . The holes size are $2.5 \times 2.5 \xi(0)$ and $1.5 \times 1.5 \xi(0)$. In calculating the resistance $\mathrm{R}(\mathrm{T})$ we fixe the current at constant value $\mathrm{j}=0.0001$ in dimensionless unit. The resistance at $T=T_{c}$ is denoted as normal resistance $R_{n}$. The critical temperature is determined 


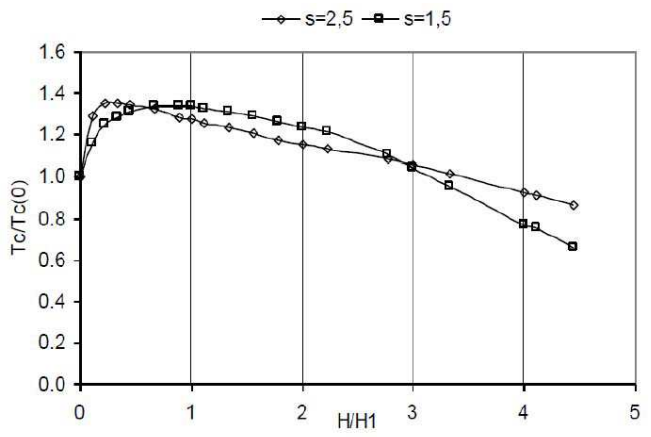

FIG. 4: Critical Temperature versus $\mathrm{H}$ for superconductor having an array of holes with a hole size of $1.5 \xi(0)^{2}$ and $2.5 \xi(0)^{2}$, symbolized respectively by different $S_{1.5}$ and $S_{2.5}$. Both have a similar lattice edge $7.5 \xi(0)^{2}$

using $R\left(T_{c}\right)=0.1 R_{n}$ [1]. The result of $R(T) / R_{n}$ versus $T_{c}(H) / T_{c}(0)$ is shown at Fig. 2. We can see from Fig. 3 the effect of magnetic field $\mathrm{H}$ at $\mathrm{H}=0$ at to the resistance. This result is just as expected in the sense that the external magnetic field tends to lower the critical temperature. The calculation indicates that at the critical temperature for superconductor having hole size $1,5 \xi(0)$ is $T_{c}(0)=0.81 T_{c}$ while for the superconductor having the hole size is $T_{c}(0)=0.82 T_{c}$

The result for $T_{c}(H)$ is determined using curve $\mathrm{R}(\mathrm{H}, \mathrm{T})$ and is presented in Fig. 4. Since the superconductor having an ar- ray of hole producing matching magnetic fields where the critical currents become maximum, we will express the magnetic field in term of $H_{n}$. It can be shown that the addition of holes lattice and exposed under magnetic field change the critical temperature up to maximum $37 \%$ than the one without magnetic field. On the superconductor having hole size $2,5 \xi(0)$ or s2,5 and s1,5 under magnetic field $0<H<3 H_{1}$ the changes are such that $T_{c}(H)>T_{c}(0)$ while at $3 H_{1} \leq H$ the changes are $T_{c}(H)<T_{c}(0)$. The first part is due to the number of vortices which is still smaller than the maximum occupancy of vortices in the hole while on the second part is the other way around. Since there is a maximum vortices trapped by holes therefore there is a bell like curve in $T_{c}(H)$. Compare to the experiment result in [1], our result fairly match in the low magnetic field. The different result obtained in high magnetic field may be due to the limitation of TDGL nature which is not so sensitive with the temperature effect.

\section{CONCLUSION}

The calculation result indicates that the addition of holes to the superconductor changes the critical temperature more significantly at lower temperature than at higher temperature. The bigger the hole sizes the changes the bigger the changes. There is still discrepancy between the calculated result using TDGL with the experiment result that may limit such calculation using TDGL.
[1] V. Silhanek, L. Van Look, R. Jonckheere, B. Y. Zhu, S. Raedts, and V. V. Moshchalkov, Phys.Rev. B 72, 014507 (2005).

[2] M. V. Milosevic and F. M. Peeters, Phys. Rev. Lett. 93, 267006 (2004).

[3] M. Kemmler, C. Gurlich, A.Sterck, H. Pohler, M. Neuhaus, M. Siegel, R. Kleiner, and, D. Koelle, Phys. Rev. Lett. 97, 147003 (2006).

[4] Harsojo, Abraha Kamsul, Nurwantoro Pekik, AIP Conference Proceedings, Vol. 1169, pp. 132-137, 2009.

[5] Tinkham, M., Introduction to Superconductivity (McGraw-Hill, New-York, 1996).

[6] Ben Xu, M. V. Milosevic, and F. M. Peeters, Phys. Rev. B 81,
064501 (2010)

[7] F. Carillo, G. Papari, D. Stornaiuolo, D. Born, D. Montemurro, P. Pingue, F.Beltram, and F.Tafuri, Phys. Rev. B 81, 054505 (2010).

[8] C. J. O.Reichhardt and C. Reichhardt and N.G.Jensen, Phys. Rev. B 63, 054510 (2001).

[9] C. Bolech, G. C. Buscaglia, and A. Lopez, Phys. Rev. B 52, R15719 (1995).

[10] Harsojo, Pekik N, Muslim, Indon. Journ .Phys., Vol. 15, No 1,15 (2004).

[11] M. Machida and H. Kaburaki, Phys. Rev. Lett. 75, 3178 (1995). 